\title{
Paraurethral Cysts in a Female Newborn
}

\author{
Shruti Dhapodkar, Kaveh Homayoon \\ Departments of Urology and Surgery, Maricopa Integrated Health Systems, Phoenix, USA \\ E-mail: Shruti.Dhapodkar@mihs.org \\ Received May 13, 2011; revised July 5, 2011; accepted July 12, 2011
}

\begin{abstract}
Paraurethral cysts are a rare congenital abnormality seen in female neonates. We present the case of a female newborn with a paraurethral cyst resulting in urinary obstruction. Surgical intervention was chosen as a result of the obstruction.
\end{abstract}

Keywords: Congenital External Genital Cyst, Paraurethral Cyst, Vaginal Cyst, Skene’s Gland, Urethra

\section{Introduction}

Congenital paraurethral cysts are rare in neonates. We present a case of a neonate with paraurethral cyst.

\section{Case Report}

The patient was a one day old female with 41 week gestational age, natural gestation and uneventful vaginal delivery who was found to have a $0.5 \times 1 \mathrm{~cm}$ protruding mass in the vaginal vestibule upon physical examination. The mass was ovoid in shape and was yellow-orange in color (Figure 1). The patient had urinary obstruction and it was suspected that the cyst was blocking the distal meatus.

An ultrasound was ordered which revealed bilateral fullness in the renal pelves. There was no evidence of hydronephrosis, nephrolithiasis or perinephric fluid.

Due to the obstruction, surgical intervention was chosen. A longitudinal incision was made over the protruding cyst, resulting in the exudation of a white milky liquid. The entire cyst was excised and unroofed.

The pathology of the cyst showed benign squamous inclusion cyst, consistent with the histology of a paraurethral cyst. The patient's urinary symptoms resolved and follow up visits showed no recurrence.

\section{Discussion}

Paraurethral glands, or Skene's glands, first described by Scottish gynecologist Alexander Skene in 1880 [1], are the largest of the female urethral glands. The paraurethral glands have 6 - 30 ducts which merge into two main ducts draining into distal urethra [2]. Paraurethral glands secrete a mucus-like substance that provides lubrication to the urethral meatus, particularly in response to sexual stimulation [3]. As such, the paraurethral glands are regarded as rudimentary analogues to the male prostate [2].

Paraurethral gland cysts are rare congenital abnormality which present as an interlabial cystic mass in newborn girls. The true incidence of this anomaly is not known. The reported incidence varies from 1 in 2074 to 1 in 7242 female births [4].

\subsection{Diagnosis}

Differential diagnoses for interlabial masses include Gartner's duct cysts, inclusion cysts of the vaginal wall, urethral neoplasm, urethral prolapse, urethral diverticulum, Mullerian remnant cyst, prolapsed ectopic ureterocele, hymenal cyst and vaginal neoplasm [5]. Usually physical examination of external genitalia is diagnostic but ultrasound can be used to facilitate in narrowing down the differentials.

\subsection{Etiology}

The exact etiology of paraurethral cysts is not known. Exposure to maternal estrogen has been reported as a possible cause for development of paraurethral cyst but there is no documented report of maternal exposure in any of the reported cases in the English literature [6]. Indeed, no reports of paraurethral cysts have been found in newborn girls born from mothers who were exposed to Diethylstilbestrol (DES), which was used for prevention of miscarriage between 1938 and 1971 [7,8]. In addition, many studies have shown an increased incidence of maternal exposure to environmental estrogens with subse- 


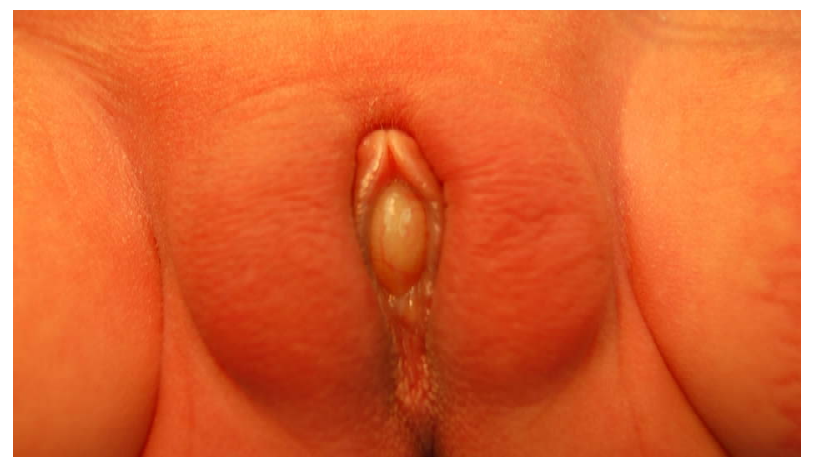

Figure 1. Paraurethral cyst presenting as a $0.5 \mathrm{~cm} \times 1 \mathrm{~cm}$ protruding mass.

quent development of congenital anomalies in their infants $[9,10]$. If maternal estrogen was a key player in the development of paraurethral cysts, we would have expected to see an increase in the incidence of paraurethral cysts. However, no such increase has been observed.

Another proposed cause for the cysts is the obstruction of Skene's gland ducts due to an improperly timed or delayed opening; stenosis of the duct; or obstruction by a mucus plug $[11,12]$.

A third theory postulates that a dislocation of the urothelium from the urogenital sinus into the neighboring area may underlie the etiology of the paraurethral cyst $[11,12]$.

\subsection{Prognosis and Treatment Options}

Many reports in the literature indicate spontaneous resolution of paraurethral cysts within 76 to 304 days [7]. Therefore, many advocate the conservative ("watch and wait") method as the primary approach for the management of congenital paraurethral cyst. It is not clear if this spontaneous resolution is as a result of gradual opening of the duct, perforation of the cyst or absorption of the cyst content.

There are also reports in the literature indicating persistence of the cyst requiring surgical drainage at a later time [6]. Drainage of the cyst by aspiration, unroofing and marsupialization have been tried with immediate resolution of the cyst. All of these procedures are safe and can be performed in a short period of time. Such an approach will reduce the need for follow up and alleviates the parents' anxiety.

\section{Conclusions}

The incidence of paraurethral cysts may be higher than reported. It is typically diagnosed upon physical examination, although ultrasound may be used to confirm. Due to many reported incidents of spontaneous resolution of these cysts within 304 days, there is a precedent for non-intervention. For persistent cysts or cases where intervention is required, drainage of the cysts by aspiration, unroofing and marsupialization have all been tried with success.

\section{References}

[1] A. J. C. Skene, "The Anatomy and Pathology of Two Important Glands of the Female Urethra," American Journal of Obstetrics \& Gynecology, Vol. 13, 1880, pp. 265-270.

[2] S. L. Tepper, L. Jagirdar, D. Heath, et al., "Homology between the Female Paraurethral (Skene's) Glands and the Prostate. Immunohistochemical Demonstration," Archives of Pathology \& Laboratory Medicine, Vol. 108, 1984, pp. 423-425.

[3] P. Merlob, C. Bahari, E. Liban, et al., "Cysts of the Female External Genitalia in the Newborn Infant," American Journal of Obstetrics \& Gynecology, Vol. 132, 1978, pp. 607-610.

[4] T. Fujimoto, T. Suwa, N. Ishii, et al., "Paraurethral Cyst in Female Newborn: Is Surgery Always Advocated?" Journal of Pediatric Surgery, Vol. 42, No. 2, 2007, pp. 400-403. doi:10.1016/j.jpedsurg.2006.10.030

[5] A. R. Nussbaum and R. L. Lebowitz, "Interlabial Masses in Little Girls: Review and Imaging Recommendations," American Journal of Roentgenology, Vol. 141, No. 1, 1983, pp. 65-71.

[6] J. E. Wright, "Paraurethral (Skene’s Duct) Cysts in the Newborn Resolve Spontaneously,” Pediatric Surgery International, Vol. 11, 1996, pp. 191-192. doi:10.1007/BF00183766

[7] L. Titus-Ernstoff, R. Troisi, E. E. Hatch, et al., "Birth Defects in the Sons and Daughters of Women Who Were Exposed in Utero to Diethylstilbestrol (DES)," International Journal of Andrology, Vol. 33, No. 2, 2010, pp. 377-384. doi:10.1111/j.1365-2605.2009.01010.x

[8] R. Clark, "Estrogen \& Breast Cancer Risk: Factors of Exposure,” BCERF Environmental Risk Factor Database.

[9] R. R. Newbold, E. Padilla-Banks and W. N. Jefferson, "Environmental Estrogens and Obesity," Molecular \& Cellular Endocrinology, Vol. 304, No. 1-2, 2009, pp. 84-89. doi:10.1016/j.mce.2009.02.024

[10] J. G. Blaivas, V. M. Pais and A. B. Retik, "Paraurethral Cysts in Female Neonate,” Urology, Vol. 7, No. 5, 1976, pp. 504-507. doi:10.1016/0090-4295(76)90191-6

[11] H. M. Kimbrough and E. D. Vaughan, "Skene's Duct Cyst in a Newborn: Case Report and Review of the Literature,” Journal of Urology, Vol. 117, 1977, pp. 387388.

[12] N. H. Lee and S. Y. Kim, "Skene's Duct Cysts in Female Newborns,” Journal of Pediatric Surgery, Vol. 27, No. 1, 1992, pp. 15-17. doi:10.1016/0022-3468(92)90094-N 